\title{
The fluid dynamics of inner-core growth
}

\author{
Bruce Buffett ${ }^{\mathrm{a}, *}$, Hiroaki Matsui ${ }^{\mathrm{b}}$ \\ ${ }^{a}$ Department of Earth and Planetary Science, University of California, Berkeley, CA 94720, USA \\ ${ }^{\mathrm{b}}$ Department of Earth and Planetary Sciences, University of California, Davis, CA 95616, USA
}

\section{A R T I C L E I N F O}

\section{Article history:}

Received 2 October 2014

Received in revised form 11 March 2015

Accepted 6 April 2015

Available online 10 April 2015

\section{Keywords:}

Geodynamo

Core processes

Thermal evolution

Inner-core anisotropy

\begin{abstract}
A B S T R A C T
Aspherical growth of the inner core has been suggested as a mechanism to produce seismic anisotropy through alignment of crystal lattices. This mechanism is viable if the response to aspherical growth occurs by slow viscous deformation. The inner core can also respond by melting and solidification at the boundary if flow in the liquid core can redistribute latent heat over the surface. We use a numerical geodynamo model to quantitatively assess the process of melting and solidification, and find that the response to aspherical growth occurs primarily through phase transitions when the viscosity of the inner core is $10^{21}$ Pa s or higher. A lower inner-core viscosity favors viscous adjustment, but the associated stresses may be too low to produce substantial crystal alignment. Independent of the primary relaxation mechanism, we expect a persistent and large-scale flow of the liquid core over the surface of the inner core. The predicted flow should be large enough to affect the crystal orientation of hcp-iron alloys during solidification, yet the absence of detectable seismic anisotropy in the top $60-80 \mathrm{~km}$ is suggestive. Either the mechanism of flow-induced alignment does not apply in the core or the intrinsic anisotropy of hcp iron at inner-core conditions is weak. Future seismological modeling using the predicted distribution of lattice preferred orientation might establish whether this texture is detectable with current observations.
\end{abstract}

(c) 2015 Elsevier B.V. All rights reserved.

\section{Introduction}

Cooling and solidification of the liquid iron core causes growth of the inner-core at a rate of roughly $10^{-3} \mathrm{~m} \mathrm{yr}^{-1}$ (e.g. Nimmo, 2007). Spatial variations in the growth rate are expected when latent heat and chemical impurities are not removed uniformly from the interface. One factor that contributes to aspherical growth is due to the effects of rotation on convection in the liquid core. Heat transport is promoted in the equatorial region (Zhang, 1992; Sumita and Olson, 2000), allowing preferential solidification at equator. Departures from hydrostatic equilibrium drive slow viscous flow through the interior of the inner core, which can produce alignment of the crystallographic axes of solid iron (Yoshida et al., 1996). The combination of persistent aspherical growth and viscous adjustment offers an attractive explanation for the presence of seismic anisotropy in the inner core (see Deuss, 2014, for a recent review).

Deviations in the shape of the inner core also perturb temperature in the liquid core. Even small variations in temperature are capable of driving flow because of the viscosity of liquid iron is

\footnotetext{
* Corresponding author.

E-mail address: bbuffett@berkeley.edu (B. Buffett).
}

very low (de Wijs et al., 1998; Pozzo et al., 2013). When the flow is vigorous enough to redistribute latent heat over the surface of the inner core, the boundary can adjust by melting and solidification, rather than by slow viscous flow through the interior. One way to assess the relative importance of these two mechanisms is to estimate the timescales for relaxation toward a hydrostatic equilibrium. In one end-member case relaxation occurs solely by viscous flow in the interior of the inner core. The relevant timescale is set by the inner-core viscosity and the density jump across the inner-core boundary. In the other end-member case relaxation occurs through phase changes at the inner-core boundary. Here the relaxation time depends on the size of temperature anomalies associated with a non-hydrostatic shape and the strength of fluid motion that results from these anomalies.

In this study we use a numerical geodynamo model to investigate the fluid motion induced by thermal anomalies at the innercore boundary. We quantify the transport of heat and assess the latent heat that must be removed or added to restore the boundary to its equilibrium position. The timescale for phase change is compared with the timescale for viscous relaxation (e.g. Cathles, 1975) to assess the primary adjustment mechanism. A low inner-core viscosity favors viscous adjustment, but the resulting stresses may be too small to cause alignment of iron crystals. On the other hand, a high viscosity, comparable to that proposed by Yoshida 
Table 1

List of physical properties.

\begin{tabular}{llll}
\hline Property & Symbol & Value & Units \\
\hline Bulk modulus (adiabatic) & $K_{S}$ & $1.3 \times 10^{12}$ & $\mathrm{~Pa}$ \\
Bulk modulus (isothermal) & $K_{T}$ & $1.2 \times 10^{12}$ & $\mathrm{~Pa}$ \\
Density Contrast at $r_{i}$ & $\Delta \rho$ & 600 & $\mathrm{~kg} \mathrm{~m}^{-3}$ \\
Density of Fluid (average) & $\rho$ & $10^{4}$ & $\mathrm{~kg} \mathrm{~m}^{-3}$ \\
Gravity at $r_{i}$ & $g\left(r_{i}\right)$ & 4.4 & $\mathrm{~m} \mathrm{~s}^{-2}$ \\
Gravity at $r_{o}$ & $g\left(r_{o}\right)$ & 10 & $\mathrm{~m} \mathrm{~s}^{-2}$ \\
Gruneisen parameter & $\gamma$ & 1.4 & - \\
Latent heat & $H$ & $10^{6}$ & $\mathrm{~J} \mathrm{~kg}^{-1}$ \\
Melting temperature & $T_{L}$ & 5500 & $\mathrm{~K}$ \\
Radius of inner core & $r_{i}$ & $1.22 \times 10^{6}$ & $\mathrm{~m}$ \\
Radius of outer core & $r_{o}$ & $3.48 \times 10^{6}$ & $\mathrm{~m}$ \\
Specific heat & $C_{p}$ & 800 & $\mathrm{~J} \mathrm{~K}^{-1} \mathrm{~kg}^{-1}$ \\
Thermal expansion & $\alpha$ & $10^{-5}$ & $\mathrm{~K}^{-1}$ \\
\hline
\end{tabular}

et al. (1996), favors adjustment by melting and solidification. Under these circumstance we expect a substantial reduction in preferential growth of the inner core and the associated development of elastic anisotropy would likely be suppressed.

\section{Numerical geodynamo model}

We use the geodynamo model Calypso (Matsui et al., 2014) to estimate the flow driven by a non-hydrostatic inner core. This flow is superimposed on a background convective flow, which maintains an internal magnetic field. We consider an incompressible and electrically conducting fluid in a spherical shell that rotates at constant angular velocity $\Omega$. The inner, $r_{i}$, and outer, $r_{o}$, radii of the shell are chosen to have an Earth-like geometry (see Table 1). Convection is driven by an unstable temperature difference, $\Delta T$, between the top and bottom boundaries. Allowing for small thermal anomalies on the bottom boundary modifies the convective flow to account for the influence of a non-hydrostatic inner core (see Section 3).

The equations for temperature, $T$, velocity, $\mathbf{V}$, and magnetic field B are written in non-dimensional form using $L=r_{o}-r_{i}$ as a characteristic length scale and $L^{2} / v$ as the characteristic timescale, where $v$ is the kinematic viscosity. Temperature and velocity are scaled by $\Delta T$ and $v / L$, respectively, while the magnetic field is scaled by $\sqrt{\rho \mu \Omega \eta}$, where $\rho$ is the fluid density, $\mu$ is the magnetic permeability and $\eta$ is the magnetic diffusivity. The resulting governing equations are specified by four dimensionless parameters

$E=\frac{v}{\Omega L^{2}}, \quad P r=\frac{v}{\kappa}, \quad P m=\frac{v}{\eta}, \quad R a=\frac{\alpha g\left(r_{o}\right) \Delta T L}{\nu \Omega}$

which include the Ekman number, E, the Prandtl number, $P r$, the magnetic Prandtl number, $P m$ and a modified Rayleigh number, $R a$. Here $\alpha$ is the coefficient of thermal expansion, $g(r)$ is the radially dependent gravity and $\kappa$ is the thermal diffusivity. The mantle is assumed to be electrically insulating, whereas the inner core $\left(r<r_{i}\right)$ can be either insulating or electrically conducting with the same conductivity as the fluid. We find that the electrical properties of the inner core have only a small influence on the flow.

\subsection{Thermal boundary conditions}

Temperature anomalies $\delta T$ on the bottom boundary are associated with radial displacements $\delta r$ of the inner-core surface. This correspondence is defined in terms of the local geotherm, $T_{A}$, and the melting temperature, $T_{L}$ (see Fig. 1 ). The boundary temperature is constrained to remain on the melting curve when the interface is shifted in radius. A positive $\delta r$ gives a boundary temperature below the average value of $T_{A}$ at the same depth. Conversely, a negative $\delta r$

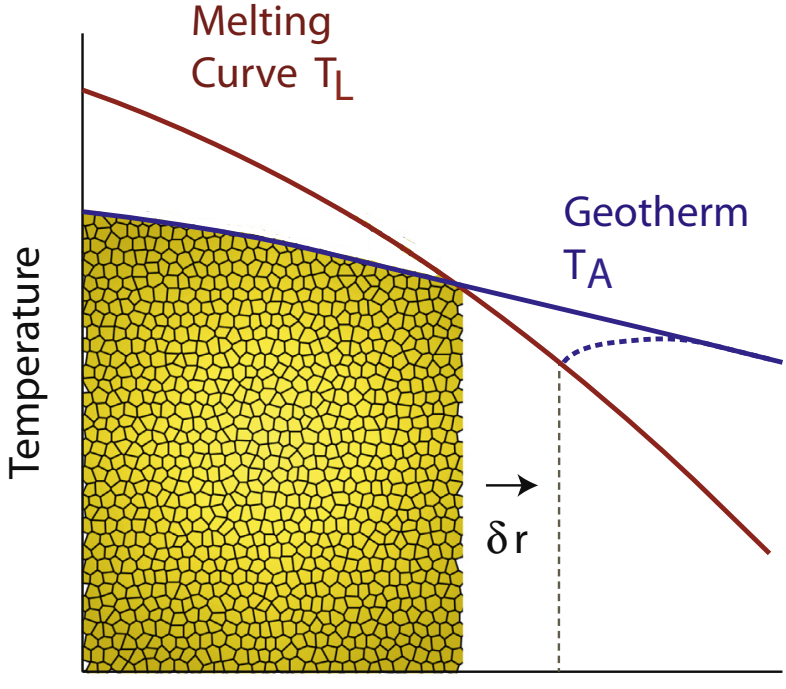

Radius

Fig. 1. Schematic illustration of thermal structure near the inner-core boundary. The intersection of the geotherm, $T_{A}$, and the melting temperature, $T_{L}$ define the location of the inner-core boundary. When a radial displacement, $\delta r$, shifts the location of the boundary, the resulting melting temperature lies below the average geotherm. Consequently, a positive $\delta r$ produces a negative temperature anomaly on the boundary. Conversely, a negative $\delta r$ causes a positive temperature anomaly on the boundary.

corresponds to a positive temperature anomaly. A quantitative relationship between $\delta T$ and $\delta r$ can be written as

$\delta T=-\rho g\left(r_{i}\right)\left(\frac{d T_{L}}{d P}-\frac{d T_{A}}{d P}\right) \delta r$

where the pressure derivative of $T_{L}$ is based on Lindemann's law (e.g. Stacey and Davis, 2008)

$\frac{d T_{L}}{d P}=\frac{2(\gamma-1 / 3) T_{L}}{K_{T}}$

and the pressure derivative of $T_{A}$ is given by

$\frac{d T_{A}}{d P}=\frac{\gamma T_{L}}{K_{S}}$

under the assumption that $T_{A}$ is an isentrope (e.g. Braginsky and Roberts, 1995). Other quantities in (3) and (4) include the Gruneisen parameter, $\gamma$, the isothermal and adiabatic bulk moduli, $K_{T}$ and $K_{S}$, and the fluid density, $\rho$ (see Table 1 ).

Temperature in an incompressible (Boussinesq) fluid can be viewed as a perturbation from an isentrope. Thus $\Delta T$ represents the temperature difference across the liquid core in excess of that predicted for the isentrope. As a result, the boundary conditions on $T$ require

$T=0$

at the core-mantle boundary $r=r_{o}$ and

$T=\Delta T+\delta T$

at the inner-core boundary, $r=r_{i}$. We adopt a constant value for $\Delta T$, specified by the choice of $R a$, and consider several values for $\delta T$. We also assume that the temperature anomaly has a spatial dependence of the form

$\delta T=\delta T_{2} P_{2}(\cos \theta)$

where $P_{2}(\cos \theta)$ is the Legendre polynomial of degree 2 and $\theta$ is colatitude. A similar dependence is assumed for the radial displacement 
$\delta r=\delta r_{2} P_{2}(\cos \theta)$

which is interpreted as the non-hydrostatic topography on the inner-core boundary due to aspherical growth. The influence of the non-hydrostatic topography enters the calculation solely through the boundary conditions on temperature. Once a value for $\delta T_{2}$ is adopted in a calculation, the corresponding value for $\delta r_{2}$ is given by (2). Typical choices for $\delta T_{2}$ correspond to $\delta r_{2} \approx 10^{-1} \mathrm{~m}$, which is small compared with the values for $r_{i}$ and $r_{o}$ (see Table 1). This justifies our use of spherical boundaries in the calculations. Similarly, the hydrostatic flattening of the inner core is approximately $2.4 \times 10^{-3}$ (Mathews et al., 1991), which is sufficiently small to neglect for present purposes.

\subsection{Rates of melting and solidification}

Convection in the liquid core establishes an aspherical pattern of heat flow from the inner core. Fig. 2 shows a representative example for a calculation with constant temperature on the lower boundary (i.e. $\delta T_{2}=0$ ). We find high heat flow at the equator, which promotes preferential solidification. Allowing for nonhydrostatic topography and the associated thermal anomalies on the lower boundary alters the flow in the liquid core. Eq. (2) indicates that enhanced growth at the equator is associated with negative temperature anomalies (and hence positive $\delta T_{2}$ ). Increasing $\delta T_{2}$ in the numerical model reduces the pole-to-equator variation in heat flow, which suppresses preferential solidification. While a complete description of the heat flow at the lower boundary includes many spherical harmonic components, it suffices for our purpose to focus specifically on the degree 2 part. Consequently, we denote the heat flow by

$q=q_{0}+q_{2} P_{2}(\cos \theta)$

where $q_{0}$ is the spherical average and $q_{2}$ represents the degree-two part of the aspherical component.

Both $q_{0}$ and $q_{2}$ are altered by changes in the non-hydrostatic topography. A modest (1\%) increase in $q_{0}$ indicates that the total heat flow increases when temperature anomalies are added to the lower boundary. However, a much larger change is observed for $q_{2}$. Increasing $\delta T_{2}$ lowers the magnitude of $q_{2}$, implying a more uniform heat flow from the inner core. A sufficient increase in $\delta T_{2}$ (and hence boundary topography) would completely eliminate the
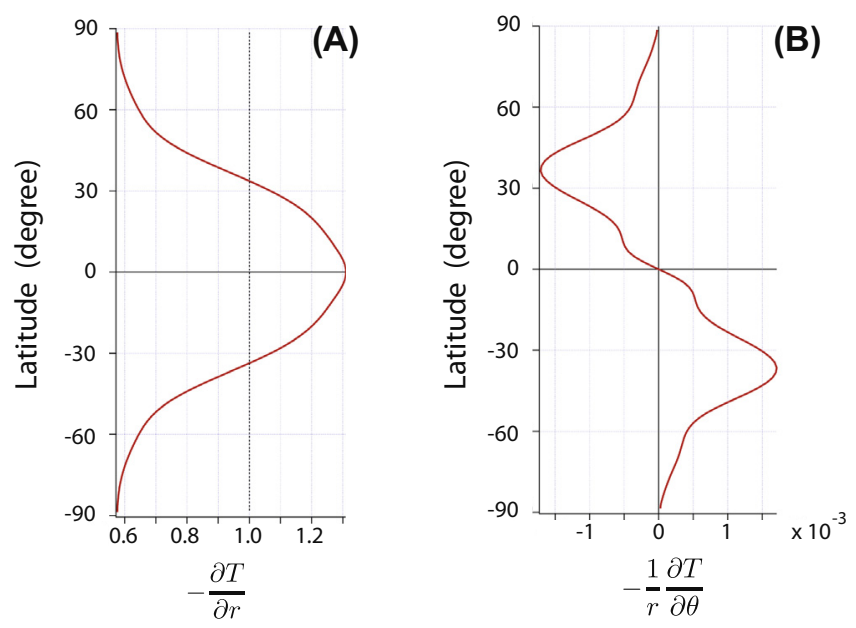

Fig. 2. Variations in the dimensionless heat flow over the surface of the inner core. (A) The radial component of heat flow is largest at the equator, which enhances the rate of solidification. (B) The meridional component of heat flow is very small, implying that heat is primarily conducted in the radial direction. aspherical part of the heat flow. Once the required topography is established the inner core would continue to grow at a constant rate over the entire surface. Whether this state is physically realized depends on the timescale for melting and solidification. Adjustments through phase changes are more likely when the timescale is short compared with the viscous adjustment time.

Suppose that we impose $\delta r$ and $\delta T_{2}$ on the inner core. How long would it take for melting and solidification to erase the imposed boundary topography? We know that the fluid motion induced by $\delta T_{2}$ alters the heat flow across the inner-core boundary. This change in heat flow either supplies or removes latent heat from the boundary, causing the interface to adjust. Conservation of energy requires

$\Delta \dot{r}=\frac{\Delta q}{\rho H}$

where $\Delta \dot{r}$ is the change in the growth rate, $\Delta q$ is the change in heat flow, and $H$ is the latent heat of solidification. The change associated with $\Delta q_{0}$ alters the average radial growth rate, whereas $\Delta q_{2}$ is responsible for removing the topography. The total amount of latent heat needed to remove the topography is $\rho H \delta r_{2}$, so the relaxation time is given by

$\tau_{p}=\frac{\rho H \delta r_{2}}{\Delta q_{2}}$

Melting and solidification should be the primary relaxation mechanism when $\tau_{p}$ is less than the timescale for viscous adjustment. An estimate for $\tau_{p}$ is obtained as follows. We impose $\delta T_{2}$ in a geodynamo model and compute the time-averaged value for $q_{2}$ at the inner-core boundary. The change in $q_{2}$ from the reference case with $\delta T_{2}=0$ defines $\Delta q_{2}$ in (11). To proceed we convert non-dimensional quantities from the geodynamo model to dimensional quantities.

\subsection{Scaling the geodynamo solution}

There are a number of ways to extract dimensional quantities from the output of a geodynamo model. Two factors guide our choice. First, we adopt a realistic value for the magnetic diffusivity to give a reasonable estimate for the magnetic-field strength. Second, we seek to preserve key parts of the force balance in the fluid outer core, particularly the geostrophic balance between thermally induced density anomalies and the Coriolis force. To be specific in our discussion we consider a particular numerical solution with dimensionless parameters $E=5 \times 10^{-5}, \mathrm{Pr}=1, \mathrm{Pm}=0.5$ and $R a=1400$. Taking $\eta=1.6 \mathrm{~m}^{2} \mathrm{~s}^{-1}$ gives $v=0.5 \eta=0.8 \mathrm{~m}^{2} \mathrm{~s}^{-1}$. The characteristic timescale is roughly $L^{2} / v=200 \mathrm{kyr}$ and the velocity scale is $v / L=3.54 \times 10^{-7} \mathrm{~m} \mathrm{~s}^{-1}$. Numerical calculations for the reference case $\left(\delta T_{2}=0\right)$ yield a dimensionless root-meansquared velocity of 180 , so the corresponding dimensional velocity is $0.64 \times 10^{-4} \mathrm{~m} \mathrm{~s}^{-1}$. This velocity is within an order of magnitude of estimates at the core surface (Holme and Olsen, 2006).

An estimate for temperature is obtained from the definition of the Rayleigh number. This particular choice preserves the thermal wind balance between temperature anomalies and the Coriolis force (e.g. Pedlosky, 1987). Setting $\alpha=10^{-5} \mathrm{~K}^{-1}, g\left(r_{o}\right)=10 \mathrm{~m} \mathrm{~s}^{-2}$ and $\Omega=0.73 \times 10^{-4} \mathrm{~s}^{-1}$ in the definition of the Rayleigh number gives $\Delta T=0.36 \mathrm{mK}$ for $R a=1400$. Comparable temperature anomalies have been inferred from geomagnetic observations (Bloxham and Jackson, 1990).

The final step is to recover an estimate of the heat flow across the lower boundary using Fourier's Law

$q=-\left(k \frac{\Delta T}{L}\right) \frac{d T}{d r}$ 
where $d T / d r$ refers to the dimensionless temperature gradient from the geodynamo solution and $k=\rho C_{p} \kappa$ is the thermal conductivity. The expression in brackets in (12) converts the dimensionless temperature gradient into a dimensional heat flow. Noting that $\kappa=v / P r$, we obtain a conversion factor of $1.2 \mathrm{~mW} \mathrm{~m}^{-2}$ using the other parameter values listed in Table 1.

\section{Thermal relaxation of boundary topography}

A series of numerical calculations are used to evaluate the relaxation time for different values of $\delta T_{2}$ (or equivalently $\delta r$ ). A reference case with $\delta T_{2}=0$ was run for several magnetic diffusion times to establish a statistical steady state. The time-averaged temperature gradient from this calculation confirms the excess heat flow at the equator of the inner core (see Fig. 2). Three other numerical solutions are run from a common initial condition using $\delta T_{2}=0.05 \Delta T, 0.1 \Delta T$ and $0.2 \Delta T$; the boundary topography, based on (2), is $\delta r_{2}=0.1 \mathrm{~m}, 0.2 \mathrm{~m}$ and $0.4 \mathrm{~m}$. The temperature gradient at the inner-core boundary converges to a steady time-average after 0.2 magnetic diffusion times or about 18 overturn times. A short transient is expected because changes in the convective flow are confined to a thin region near the inner core.

Fig. 3 shows the time-averaged zonal flow immediately above the inner-core boundary when $\delta T_{2}=0.1 \Delta T$. The azimuthal component of flow, $V_{\phi}$, is driven by the imposed horizontal temperature gradient between the equator and pole. The direction and magnitude of this flow are consistent with a thermal wind balance. The presence of frictional forces in the boundary region (Batchelor, 1967, p. 199) causes a poleward flow, $V_{\theta}$, which carries cold fluid from the equator to the poles. A broad return circulation brings warm fluid from the poles back toward the equator.

Changes in the thermal structure above the inner core are shown in Fig. 4. Here we compare the time-averaged zonal temperature for cases $\delta T_{2}=0$ and $\delta T_{2}=0.1 \Delta T$. Both calculations have closely spaced isotherms in the equatorial region, consistent with high heat flow. A weaker heat flow is evident in the polar region. The aspherical calculation has a higher (imposed) boundary temperature at the poles, but cooler temperatures in the overlying fluid. Thus the polar heat flow is enhanced by the introduction of thermal anomalies associated with aspherical structure. This outcome leads to a reduction in the pole-to-equator heat flow variation, which lowers the rate of aspherical growth.

A quantitative assessment of the change in heat flow is shown in Fig. 5. Typical values for $\Delta q_{2}$ are on the order of few $\mathrm{mW} \mathrm{m}^{-2}$, but there is a steady increase in heat flow with the amplitude of $\delta T_{2}$. Simple predictions based on a thermal wind balance give a
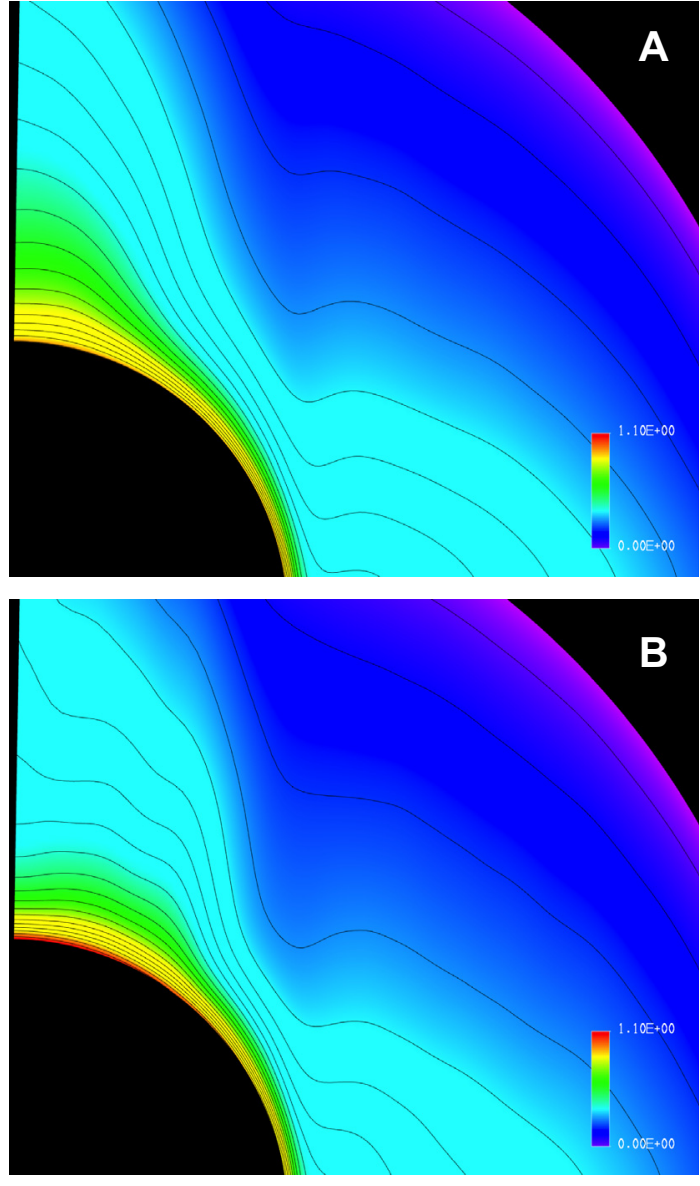

Fig. 4. Thermal structure above the inner core in (A) the reference calculation with $\delta T_{2}=0$ and (B) the perturbed calculation with $\delta T_{2}=0.1 \Delta T$. Closely spaced isotherms in the equatorial region are consistent with a higher heat flow. A warmer boundary temperature in the polar region of the perturbed calculation coincides with a steeper temperature gradient in the overlying fluid. This means that the flow induced by the boundary temperature anomaly reduces the pole-to-equator variations in heat flow. The thickness of the thermal boundary layer at midlatitudes is roughly $0.06 \mathrm{~L}$.

linear relationship between $\Delta q_{2}$ and $\delta T_{2}$ (see below). Thus deviations from this linear trend indicate complications in the dynamics, possibly due to nonlinear interactions between the boundary-driven flow and the background convection. Estimates for the timescale $\tau_{p}$ in (11) are shown in Fig. 5B. There is a weak tendency

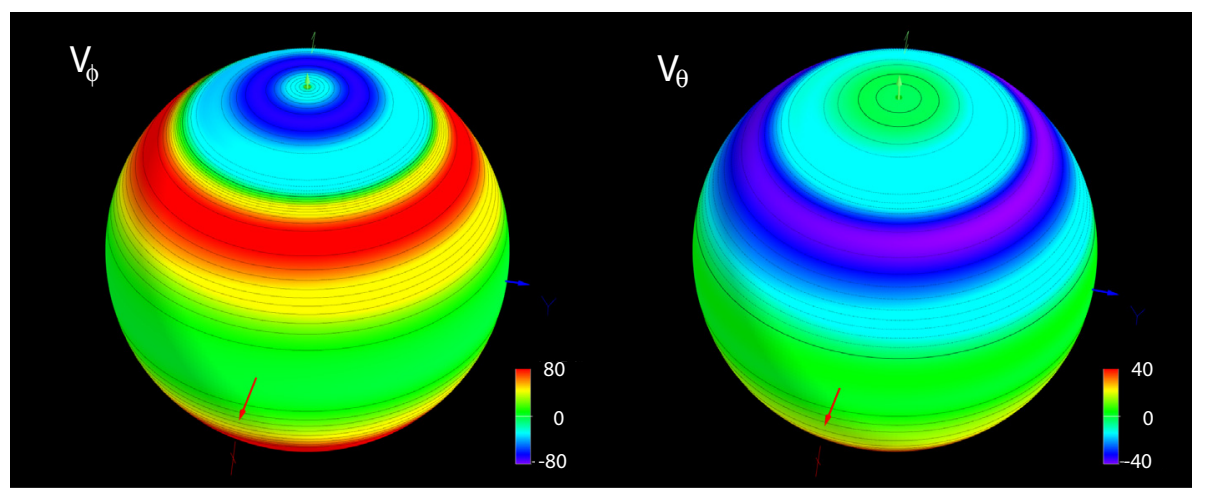

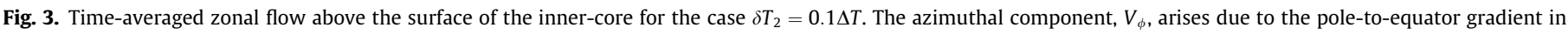

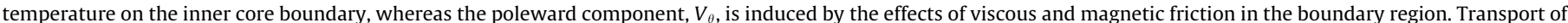

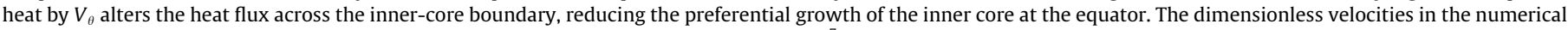
model are converted to dimensional values using the characteristic scale $v / L=3.54 \times 10^{-7} \mathrm{~m} \mathrm{~s}^{-1}$. 

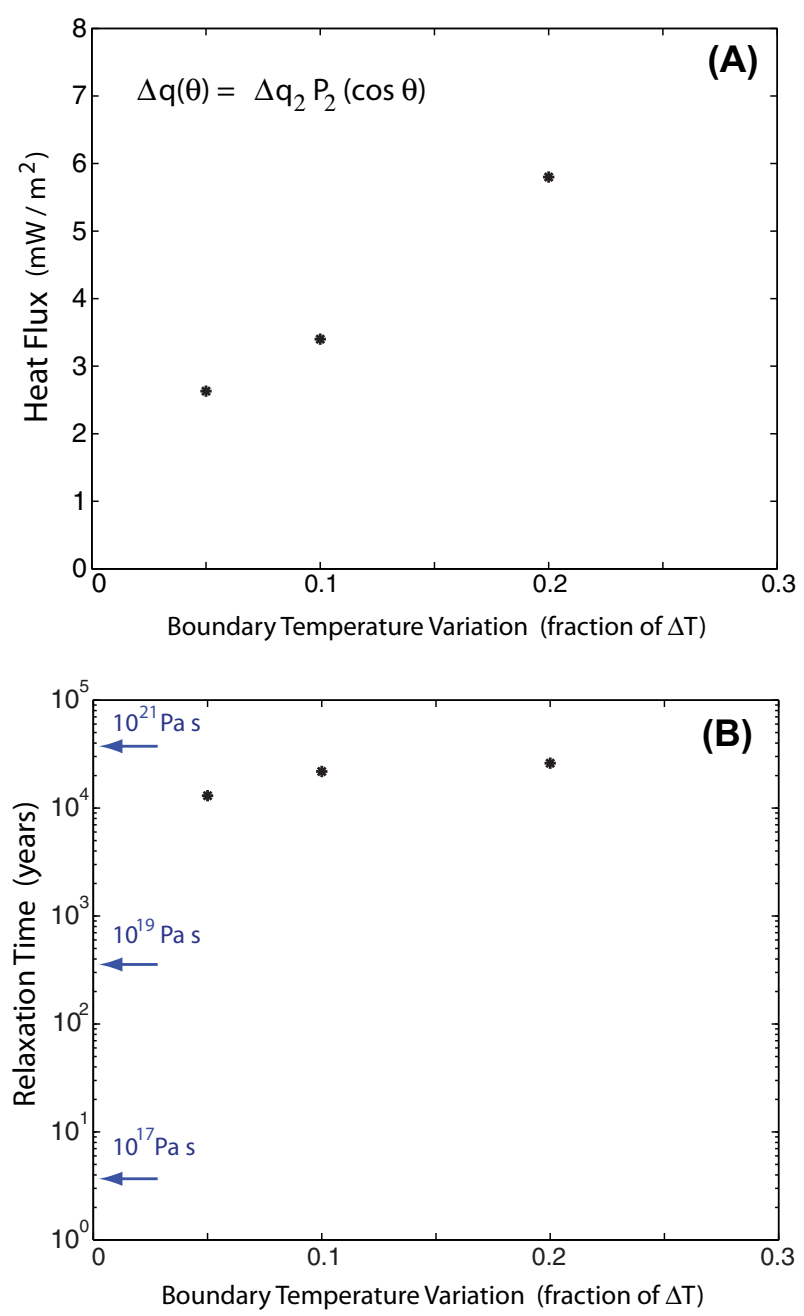

Fig. 5. (A) Change in the degree-two part of the heat flow, $\Delta q_{2}$, at the inner core boundary as a function of the boundary temperature anomaly, $\delta T_{2}$. Increasing $\delta T_{2}$ (and hence $\delta r$ ) reduces the pole-to-equator variation in heat flow, corresponding to a larger change from the reference case. (B) The relaxation time for melting and solidification, $\tau_{p}$, computed from (11). The arrows indicate viscous relaxation times for various choices of inner-core viscosity.

for $\tau_{p}$ to increase with the amplitude of $\delta T_{2}$, consistent with departures from a linear relationship between $\Delta q_{2}$ and $\delta T_{2}$. We obtain a relaxation time of $13 \mathrm{kyr}$ at $\delta T_{2}=0.05 \Delta T$ and $\tau_{p}=26 \mathrm{kyr}$ for $\delta T_{2}=0.2 \Delta T$. The intermediate value at $\delta T_{2}=0.1 \Delta T$ gives $\tau_{p}=21.8 \mathrm{kyr}$.

Thermal relaxation of the boundary also occurs when the surface is displaced by a lateral translation of the inner core. This type of disturbance is relevant for the recently proposed translational mode of convection (Alboussière et al., 2010; Monnereau et al., 2010). We do not consider the full dynamics of translational convection, but simply extend the preceding analysis to quantify the thermal relaxation process. An imposed displacement in the equatorial plane can described by

$\delta r=\delta r_{1}^{1} Y_{1}^{1}(\theta, \phi)$

where $Y_{l}^{m}(\theta, \phi)$ is a spherical harmonic of degree of degree $l$ and order $m$. The associated temperature anomaly, $\delta T_{1}^{1}$, modifies the flow in the outer core, causing a change in the heat flow across the inner-core boundary. The $Y_{1}^{1}$ part of the time-average heat flow vanishes in the reference case $\left(\delta T_{1}^{1}=0\right)$, so the change is heat flow due to non-zero $\delta T_{1}^{1}$ is simply equal to the $Y_{1}^{1}$ part of the heat flow in the perturbed calculation. We denote this change in heat flow by
$\Delta q_{1}^{1}$ and use the corresponding $\delta r_{1}^{1}$ in (11) to define a relaxation time. Calculations with $\delta T_{1}^{1}=0.05 \Delta T, 0.1 \Delta T$ and $0.2 \Delta T$ give a remarkably consistent estimate of $\tau_{p}=21 \pm 0.5 \mathrm{kyr}$. This value is within the range of estimates given previously for aspherical growth of the inner core, although the details of the flow in the outer core are somewhat different (see below).

The study of Alboussière et al. (2010) used dimensional arguments to estimate the transport of latent heat by convection in the outer core. An order-of-magnitude estimate for the heat flow is

$\Delta q_{1}^{1}=\rho v^{\prime} C_{p} \delta T_{1}^{1}$

where $v^{\prime}$ is a representative velocity in the liquid core. Comparisons with the output from the geodynamo model suggest that (14) over estimates the heat flow when $v^{\prime}$ is approximated using the rootmean-square (rms) velocity. However, better agreement is found when $v^{\prime}$ is about a factor of ten smaller than the rms velocity.

\subsection{Application to the Earth's core}

The relevance of the preceding estimates depends on the validity of the numerical model. Computational limitations prevent these models from reproducing all aspects of the dynamics, but it is possible to reliably capture some aspects of the flow. For example, the zonal flow, $V_{\phi}$, in the numerical model represents a thermal wind, which is consistent with expectations for the Earth's core (Bloxham and Gubbins, 1987). The usual form of the thermal wind equation can be derived from a balance between the Coriolis force and a local hydrostatic pressure (Pedlosky, 1987). In a thin layer, where only the radial component of the rotation vector is retained in the dynamics, the thermal wind balance becomes

$f \frac{\partial V_{\phi}}{\partial r}=\alpha g\left(\frac{1}{r} \frac{\partial T}{\partial \theta}\right)$

where $f=2 \Omega \cos \theta$. The assumption of a thin layer is appropriate here because the flow induced by boundary topography is confined to the vicinity of the inner core. The thermal wind equation in (15) can also be derived from a balance between the Coriolis and buoyancy terms in the $\phi$-component of the vorticity equation, without explicitly invoking a hydrostatic assumption. Writing (15) in nondimensional form involves only one dimensionless parameter, the modified Rayleigh number $R a$. This dependence is the main reason for evaluating the temperature scale $\Delta T$ from the definition of $R a$. Our choice ensures a realistic relationship between the boundary temperature anomalies and the resulting flow.

The poleward component of flow is described by the $\theta$-component of the vorticity equation. This component of flow is not possible without resistive or frictional forces in the boundary region because the density gradient vanishes in the $\phi$ direction. Retaining the effects of magnetic and viscous forces in the vorticity equation yields

$f \frac{\partial V_{\theta}}{\partial r}=\frac{B_{r}}{\rho \mu}\left(\frac{\partial^{2} B_{\phi}}{\partial r^{2}}\right)+v\left(\frac{\partial V_{\phi}}{\partial r^{3}}\right)$

where the horizontal gradients in $\mathbf{V}$ and $\mathbf{B}$ are assumed to be small relative to the radial gradients on account of the thin-layer approximation. When $B_{\phi}$ is produced from $B_{r}$ by shear in the thermal wind, the $\phi$ component of the steady induction equation requires

$\eta \frac{\partial B_{\phi}^{2}}{\partial r^{2}} \approx-B_{r} \frac{\partial V_{\phi}}{\partial r}$

Substituting (17) in (16) gives

$2 \cos \theta \frac{\partial V_{\theta}}{\partial r}=-\left(\frac{B_{r}^{2}}{\rho \mu \eta \Omega}-\frac{v}{\Omega} \frac{\partial^{2}}{\partial r^{2}}\right) \frac{\partial V_{\phi}}{\partial r}$ 
where we have separated the terms in $f=2 \Omega \cos \theta$. The first term on the right-hand side represents the magnetic force, which can be written more concisely in terms of the dimensionless radial magnetic field near the inner-core boundary (denoted for present purposes as $\left.\tilde{B}_{r}=B_{r} / \sqrt{\rho \mu \eta \Omega}\right)$. A representative value at midlatitudes is $\tilde{B}_{r} \approx 0.5$. The second term on the right-hand side of (18) represents the viscous force. Outside the viscous boundary layer the shear $\partial V_{\phi} / \partial r$ is set by the thermal wind equation in (15). We expect this shear to be constant in a hypothetical case where the horizontal temperature gradient does not vary with radius. Consequently, radial variations in the shear outside the viscous boundary layer depend on radial variations in the temperature gradient. When the thermal structure around the inner core is confined to a thin layer with thickness $\epsilon L$, the radial variations in shear can be characterized by a comparable length scale. With this assumption the ratio of magnetic to viscous forces is approximated by $\left(\epsilon \tilde{B}_{2}\right)^{2} E^{-1}$. For $E=5 \times 10^{-5}$ and $\epsilon \approx 0.06$ at mid-latitudes, the ratio of magnetic to viscous forces is about 20 . As long as the thickness of the thermal halo around the inner core is large compared with the depth of the viscous boundary layer $\left(l_{V}=E^{1 / 2} L\right)$, the transport of heat toward the poles should be dominated by the influence of magnetic forces. Scaling the geodynamo solution with a realistic value for $\eta$ appears to give a reasonable estimate for the magnetic field (and hence $V_{\theta}$ ). Consequently, we expect a reasonable estimate for the resulting heat transport.

One possible complication involves the heat transported by flow inside the viscous boundary layer. Because the core viscosity is too large in the numerical calculation, it is likely that the poleward flow in the viscous boundary layer is over estimated. To assess the significance of this effect, we integrate the thermal wind equation from the top of thermal layer, where horizontal temperature gradients vanish, to the top of the viscous boundary layer. The resulting velocity defines a "free-stream" value just above the viscous boundary layer. An order-of-magnitude estimate is

$V_{\phi}\left(r_{i}\right)=-\left(\frac{\alpha g \epsilon L}{f r_{i}}\right) \frac{\partial T}{\partial \theta}$

At mid-latitudes $\partial T / \partial \theta=-3 \delta T_{2} / 2$, so (19) gives an eastward flow, consistent with that shown in Fig. 3 . Note that $V_{\phi}\left(r_{i}\right)$ depends linearly on $\delta T_{2}$. Integrating the poleward transport through the viscous boundary layer gives (Batchelor, 1967)

$\int_{0}^{l_{V}} V_{\theta} d r=\frac{1}{2} V_{\phi}\left(r_{i}\right) E^{1 / 2} L$

which is smaller than the integrated poleward flow above the viscous boundary layer

$\int_{l_{V}}^{\epsilon L} V_{\theta} d r \approx \frac{1}{2} V_{\phi}\left(r_{i}\right) \tilde{B}_{r}^{2} \epsilon L$

Consequently, we do not expect the choice of Ekman number in the numerical calculations to substantially affect the heat transport over the surface of the inner core.

\subsection{Comparison with viscous relaxation time}

Melting and solidification at the inner-core boundary is the primary relaxation mechanism when $\tau_{p}$ is shorter than the timescale for viscous adjustment. The latter depends on the inner-core viscosity, $v_{s}$, the density contrast, $\Delta \rho$, between the solid and overlying fluid and wavelength of the boundary topography. For degree-two boundary topography in (8), the adjustment time in a uniform, viscous sphere is (Cathles, 1975)

$\tau_{v}=\frac{3.8 \rho_{s} v_{s}}{\Delta \rho g\left(r_{i}\right) r_{i}}$ where $\rho_{s}$ is the density of the solid. The product $\rho_{s} v_{s}$ defines the dynamic viscosity (in Pa $s$ ), which is more commonly quoted in studies of inner-core deformation (e.g. Yoshida et al., 1996; Deguen et al., 2011).

A transition in relaxation mechanisms is expected when $\tau_{p}$ and $\tau_{v}$ are comparable. We previously obtained $\tau_{p}=13-26 \mathrm{kyr}$ for the case of aspherical growth. A comparable timescale for viscous relaxation is predicted with a dynamic viscosity of 3.7$6.9 \times 10^{20}$ Pa s. Smaller viscosities favor viscous relaxation, whereas higher values promote melting and solidification.

The study of Yoshida et al. (1996) proposed a viscosity of $10^{21} \mathrm{~Pa}$ s to sustain meter-scale boundary topography. Adopting a lower viscosity reduces the boundary topography and lowers the associated stresses. A recent (upward) revision in the rate of inner-core growth (e.g. Hirose et al., 2013) lowers the required viscosity, but not by more than a factor of four. Consequently, the viscosity needed to produce crystal alignment may be too large to favor viscous flow as the primary relaxation mechanism. Instead, the boundary adjustment should occur mainly by melting and solidification, although the two rates of adjustment are fairly close with a viscosity of $10^{21} \mathrm{~Pa} \mathrm{~s}$ (corresponding to $\tau_{v}=37 \mathrm{kyr}$ ).

The net effect of melting and solidification is a reduction in the rate of preferential growth of the inner core and the associated viscous deformation. These conclusions are reinforced if the boundary topography has a shorter wavelength because the strength of thermal wind increases with larger horizontal temperature gradients. As a result, the timescale for phase change decreases, whereas the timescale for viscous adjustment increases.

A further complication arises if the inner core is thermally stratified. Boundary topography due to aspherical growth of the inner core displaces surfaces of constant density in the interior, which act to oppose a sustained flow (Buffett and Bloxham, 2000; Deguen et al., 2011). In effect, the boundary topography is maintained isostatically by density anomalies in the interior. However, this boundary topography is gradually eroded through melting and solidification in response to flow driven in the liquid core by temperature anomalies on the boundary. The effects of thermal stratification could be overcome if the inner core was compositionally unstable (Gubbins and Davies, 2013). The likelihood of this state depends on details of the phase diagram for the relevant liquid iron alloy in the core (Labrosse, 2014).

Viscous relaxation is also relevent for the occurrence of translational convection in the inner core (Alboussière et al., 2010; Monnereau et al., 2010). Translation is expected when $\tau_{v}$ is large, and linear stability calculations (Deguen, 2012) give a quantitative estimate of the threshold $\tau_{p} / \tau_{v}<4.4$, based on the definition of $\tau_{v}$ in (22). Consequently the condition for translational convection is not very different from the transition in primary relaxation mechanism. However, the nature of flow in the outer core due to innercore translation is fundamental different than the boundary layer flows associated preferential growth. The numerical geodynamo model predicts more vigorous convection over the warm hemisphere and weaker convection above the cooler boundary. The change in heat flow allows the topography to relax on a timescale $\tau_{p}=21 \mathrm{kyr}$. Linear stability calculations require $\tau_{v}>4.8 \mathrm{kyr}$ for translation, so the corresponding viscosity is greater than $1.3 \times 10^{20}$ Pa s. A somewhat lower viscosity was predicted by Deguen et al. (2013) using the order-of-magnitude estimate of heat flow in (14).

\section{Implications for inner-core anisotropy}

The numerical geodynamo solutions offer several insights into the origin of seismic anisotropy in the inner core. Our reference solution $(\delta T=0)$ affirms the strong influence of rotation on heat 
transport through the outer core. Heat is transferred primarily through the equatorial region, which should cause preferential solidification at the equator. However the orientation of heat flow at the inner-core boundary is almost entirely radial (see Fig. 2). If iron crystals are aligned during solidification by the direction of the local temperature gradient (Bergman, 1997) then we would not expect much deviation from a radial alignment. This orientation cannot account for the polar direction of fast seismic velocities in the inner core. On the other hand, there is little evidence for seismic anisotropy in the top $60-80 \mathrm{~km}$ of the inner core (Song and Helmberger, 1995; Ouzounis and Creager, 2001; Waszek and Deuss, 2011), so a radial alignment due to temperature gradients near the top of the inner core is compatible with these observations.

Persistent boundary topography and viscous adjustment can account for the development of seismic anisotropy, but the details depend on the primary relaxation mechanism. Yoshida et al. (1996) favored a viscosity of $10^{21}$ Pa s and meter-scale boundary topography to ensure stresses on the order of $10^{4} \mathrm{~Pa}$. This level of stress was found to be sufficient to produce detectable crystal alignment through recrystallization. If the viscosity is as high as the value proposed by Yoshida et al. (1996), then the present calculations marginally favor melting and solidification as the primary relaxation mechanism. The associated stresses might not change substantially from the estimate of Yoshida et al. (1996) because a non-hydrostatic inner-core shape is still needed to drive melting and solidification. However, the resulting strain rate of the inner core would be significantly reduced. Indeed any process of texture development that relies on strain rate rather than stress would be suppressed once melting and solidification at the boundary becomes the primary relaxation mechanism. While the relevant deformation processes in the inner core are poorly understood (e.g. Reaman et al., 2011), the role of flow in the outer core is liable to be an important factor.

Independent of the primary relaxation mechanism, the presence of boundary topography should drive a persistent flow over the surface of the inner core. Both $V_{\phi}$ and $V_{\theta}$ components are predicted near the inner-core boundary. This flow can affect crystal alignment during solidification. Field observations (Weeks and Gow, 1978) and laboratory experiments (Langhorne, 1983; Bergman et al., 2005) on sea ice have shown that the $c$-axes of hcp-ice are preferentially aligned in the direction of flow. A similar behavior is observed in hcp metal alloys (Bergman et al., 2003), although complications can arise when buoyancy-driven flow due to solute variations is superimposed on a horizontal flow. The preferred crystallographic orientation in all cases is thought to permit more efficient removal of solute from the boundary. Iron alloys in the inner core appear to have an hcp structure (Tateno et al., 2012), so similar processes should align the c-axes in horizontal planes. The strongest component of flow above the inner core is east-west, but there would also be a smaller poleward component in the northern and southern hemispheres. A largescale horizontal alignment of crystals should be detectable if the intrinsic crystal anisotropy is sufficiently strong and the degree of alignment is high.

The absence of substantial anisotropy at the top of the inner core would seem to argue against one or both of these assumptions. First-principle calculations of the properties of hcp iron currently yield divergent views on the strength of elastic anisotropy (Sha and Cohen, 2010; Martorell et al., 2013). On the other hand, the magnitude of flow in the liquid core should be large enough to have an important influence on solute transport. Even if the chemical (solute) boundary layer is only a few meters thick, a flow of roughly $10^{-5} \mathrm{~m} \mathrm{~s}^{-1}$ (see Fig. 3) should transport solute much more efficiently than diffusion, based on a nominal chemical diffusivity of $10^{-8} \mathrm{~m}^{2} \mathrm{~s}^{-1}$ (Pozzo et al., 2013). Thus it would be reasonable to expect a flow-induced crystal alignment over the surface of the inner core.

There might be several reasons why this texture is not detected. For example, the predicted crystal orientation may not be adequately sampled by current observations. Subsequent deformation might gradually tilt this initial crystal alignment into a more easily observed orientation at depth. It is also possible that the observed seismic anisotropy is not attributed to crystallographic alignment, but arises from oriented layers of melt which are not completely expelled by compaction (Sumita et al., 1996).

\section{Conclusions}

We use a numerical geodynamo model to quantitatively assess the adjustment of inner-core topography through melting and solidification. For the long wavelength topography due to aspherical growth of the inner core, we obtain relaxation times of 13-26 kyr, depending on the amplitude of topography. Comparable relaxation times by viscous flow in the inner core are obtained with an average viscosity of $3.7-6.9 \times 10^{20} \mathrm{~Pa}$. Higher values of viscosity imply that topography is eroded by melting and solidification, whereas lower values promote viscous adjustment. Shorter wavelength topography preferentially adjusts by melting and solidification because the temperature gradients (and the flow) increase with shorter wavelength. The opposite is true for viscous relaxation. A shorter wavelength increases the viscous relaxation time, roughly in proportional to the spherical harmonic degree of the topography (Cathles, 1975).

Relaxation of boundary topography by melting and solidification has several consequences for the development of seismic anisotropy in the inner core. First, we do not expect a large-scale flow through the interior of the inner core in response to aspherical growth when the viscosity is roughly $10^{21} \mathrm{~Pa} s$ or larger. In this case, melting and solidification effectively relax the topography before viscous flow can accumulate large strains. Development of crystallographic alignment through the associated deformation is also weak. Stable stratification of the inner core can also suppress viscous flow in the interior of the inner core by confining motion to the surface region (Deguen et al., 2011). However, even a surficial flow is in doubt if the timescale for viscous adjustment in a thin layer is long compared with the timescale for adjustment through phase transitions.

A second aspect of melting and solidification is the persistent flow in the outer core due the presence of boundary topography. Independent of the primary relaxation mechanism, we expect a steady and large-scale flow in the outer core, which can affect the orientation of crystals during solidification. Observations of flow-induced crystal alignment in sea ice (Weeks and Gow, 1978) and metal alloys (Bergman et al., 2003) suggest that similar processes should occur over the surface of the inner core. The amplitude of flow in the outer core is probably sufficient to have an influence on solidification, yet the absence of substantial anisotropy in the top $60-80 \mathrm{~km}$ raises questions. The anisotropy may be oriented in such a way to evade detection with current observations or the intrinsic elastic anisotropy of hcp iron is weak at inner-core conditions. Distinguishing between these possibilities would offer new insights into the origin of seismic anisotropy in the inner core.

\section{Acknowledgement}

This work is supported by the National Science Foundation. We thank Renaud Deguen and an anonymous reviewer for thoughtful comments and suggestions. 


\section{References}

Alboussière, T., Deguen, R., Melzani, M., 2010. Melting-induced stratification above the Earth's inner core due to convective translation. Nature 466, 744-747.

Batchelor, G.K., 1967. An Introduction to Fluid Dynamics. Cambridge University Press, Cambridge, UK.

Bergman, M.I., 1997. Measurement of elastic anisotropy due to solidification texturing and the implications for the Earth's inner core. Nature 389, 60-63.

Bergman, M.I., Agrawal, S., Carter, M., Macleod-Siberstein, M., 2003. Transverse solidification textures in hexagonal close-packed alloys. J. Crystal Growth 255, 204-211.

Bergman, M.I., MacLeod-Silberstein, M., Haskel, M., Chandler, B., Akpan, N., 2005. A laboratory model for solidification of Earth's core. Phys. Earth Planet. Inter. 153, 150-164.

Bloxham, J., Gubbins, D., 1987. Thermal core-mantle interactions. Nature 325, 511 513.

Bloxham, J., Jackson, A., 1990. Lateral temperature variations at the core-mantle boundary deduced from the magnetic field. Geophys. Res. Lett. 17, 1997-2000.

Braginsky, S.I., Roberts, P.H., 1995. Equations governing convection in Earth's core and the geodynamo. Geophys. Astrophys. Fluid Dyn. 79, 1-97.

Buffett, B.A., Bloxham, J., 2000. Deformation of Earth's inner core by electromagnetic forces. Geophys. Res. Lett. 27, 4001-4004.

Cathles, L.M., 1975. The Viscosity of the Earth's Mantle. Princeton University Press, Princeton, NJ.

Deguen, R., Cardin, P., Merkel, S., Lebensohn, R.A., 2011. Texturing in Earth's inner core due to preferential growth in its equatorial plane. Phys. Earth Planet. Inter $188,73-184$

Deguen, R., 2012. Structure and dynamics of Earth's inner core. Earth Planet. Sci. Lett. 333-334, 211-225.

Deguen, R., Alboussiere, T., Cardin, P., 2013. Thermal convection in Earth's inner core with phase change at its boundary. Geophys. J. Int. 194, 1310-1334.

de Wijs, G.A., Kresse, G., Vocadlo, L., Dobson, D., Alfe, D., Gillan, M.J., Price, G.D., 1998. The viscosity of liquid iron at the physical conditions of the Earth's core. Nature 392, 805-807.

Deuss, A., 2014. Heterogeneity and anisotropy of Earth's inner core. Ann. Rev. Earth Planet. Sci. 42, 103-126.

Gubbins, D., Davies, C.J., 2013. Compositional instability of Earth's solid inner core. Geophys. Res. Lett. 40, 1084-1088.

Hirose, K., Labrosse, S., Hernlund, J., 2013. Composition and state of the core. Ann. Rev. Earth Planet. Sci. 41, 657-691.

Holme, R.T. Olsen, N., 2006. Core surface flow modeling from high-resolution secular variations. Geophys. J. Int. 166, 518-528.

Labrosse, S., 2014. Thermal and compositional stratification of the inner core. C. R. Geosci. 346, 119-124.

Langhorne, P.J., 1983. Laboratory experiments on crystal alignment in $\mathrm{NaCl}$ ice. Ann. Glaciol. 4, 163-169.
Martorell, B., Brodholt, J., Wood, I.G., Vocadlo, L., 2013. The effect of nickel on the properties of iron at the conditions of Earth's inner core: ab initio calculations of seismic wave velocities of Fe-Ni alloys. Earth Planet. Sci. Lett. 365, 143-151.

Mathews, P.M., Buffett, B.A., Herring, T.A., Shapiro, I.I., 1991. Forced nutations of the Earth: influence of inner core dynamics, 2. Numerical results and comparisons. J. Geophys. Res. 96, 8243-8257.

Matsui, H., King, E., Buffett, B., 2014. Multi-scale convection in a geodynamo simulation with uniform heat flux along the outer boundary. Geochem. Geophys. Geosys. 15, 3212-3225. http://dx.doi.org/10.1029/2014GC005432.

Monnereau, M., Calvet, M., Margerin, L., Souriau, A., 2010. Lopsided growth of Earth's inner core. Science 328, 1014-1017.

Nimmo, F., 2007. Energetics of the core. Treatise on Geophysics, 8. Elsevier Science, pp. 253-303.

Ouzounis, A., Creager, K.C., 2001. Isotropy overlying anisotropy at the top of the inner core. Geophys. Res. Lett. 28, 4331-4334.

Pedlosky, J., 1987. Geophysical Fluid Dynamics. Springer-Verlag, New York.

Pozzo, M., Davies, C., Gubbins, D., Alfè, D., 2013. Transport properties for liquid silicon-oxygen mixtures at Earth's core conditions. Phys. Rev. B 87, 014110.

Reaman, D.M., Daehn, G.S., Panero, W.R., 2011. Predictive mechanism for anisotropy development in the Earth's inner core. Earth Planet. Sci. Lett. 312, 437-442.

Sha, X.W., Cohen, R.E., 2010. Elastic isotropy of epsilon-Fe under Earth's core conditions. Geophys. Res. Lett. 37, L10302. http://dx.doi.org/10.1029/ 2009GL042224.

Song, X., Helmberger, D.V., 1995. Depth dependence of anisotropy of Earth's inner core. J. Geophys. Res. 100, 9805-9816.

Sumita, I., Yoshida, S., Kumazawa, M., Hamano, Y., 1996. A model for sedimentary compaction of a viscous medium and its application to inner-core growth. Geophys. J. Int. 124, 502-524.

Stacey, F.D., Davis, P.M., 2008. Physics of the Earth, fourth ed. Cambridge University Press, Cambridge, UK.

Sumita, I., Olson, P., 2000. Laboratory experiments on high Rayleigh number thermal convection in a rapidly rotating hemispherical shell. Phys. Earth Planet. Inter. 117, 153-170.

Tateno, S., Hirose, K., Komabayashi, T., Ozawa, H., Ohishi, Y., 2012. The structure of Fe-Ni alloy in Earth's inner core. Geophys. Res. Lett. 39, L12305. http:// dx.doi.org/10.1029/2012GL052103.

Waszek, L., Deuss, A., 2011. Distinct layering in the hemispherical seismic velocity structure of Earth's upper inner core. J. Geophys. Res. 111, B12313. http:// dx.doi.org/10.1029/2011JB008650.

Weeks, W.F., Gow, A.J., 1978. Preferred crystal orientations in the fast ice along the margins of the Arctic Ocean. J. Geophys. Res. 83, 5105-5121.

Yoshida, S., Sumita, I., Kumazawa, M., 1996. Growth model of the inner core coupled with outer core dynamics and the resulting elastic anisotropy. J. Geophys. Res. 101, 28085-28103.

Zhang, K., 1992. Spiralling columnar convection in rapidly rotating spherical fluid shells. J. Fluid Mech. 236, 535-556. 